sent [rom overseas to $\mathrm{D}$ Block, but which require no longer the special supervision provided there. Cases are sent also direct]y to the section from overseas.

The type of case observed and treated in these sections is similar. They are: Most forms of functional paralysis, especially paraplegia, disturbances of speech and articulation, amnesia or loss of memory, the effects of terrifying dreams, mutism, deafness, deaf-mutism, amblyopia, "bentbask," tremblings and motor agitations, tic-like movements, sleeplessness, nervous debility, indecision, loss of selfconfidence, and the milder forms of neurasthenia, simple mental confusion, the anxiety psycho-neuroses, and simple mental depression.

The treatment adopted consists chiefly of rest and feeding ; massage and electrical applications in suitable cases; baths when these seem indicated; and psychotherapy in the form of simple suggestion and occasional hy pnosis.

In a general way the results of treatment at the Fourth London General Hospital show 40 per cent. of cases returned to light duty, 20 per cent. invalided, and 20 per cent. trans ferred for further treatment to the special institutions.

2. The Speoial Institutions - the Red Cross Military Hospital, Magnull, and the Springfield Wur Hospital.-Buth these hospitals are constructed on somewhat similar lines in that they are provided with single rooms and special accommodation for cases requiring isolation and supervision in addition to day rooms and dormitories. The Maghull Military Hospital had not been used for the treatment of patients before it was taken over by the War Office, but the Springfield War Hospital had been employed as a hospital for defective children for about ten years.

The available accommodation in the two hospitals amounts to about 550 beds. No case is admitted directly from overseas to either of these institutions, as all cases have received a course of treatment at one of the Military Hospitals at home before transference. The patients most suitable for treatment in these institutions are cases of neurasthenia of a severe or protracted character, the milder psychoses, such as simple melancholia and the anxiety psychoses, psychoses with obsessions and fears, profound amnesia, epilepsy, highgrade mental defectives, the milder types of primary dementia, and all cases of a functional character which do not lend themselves to treatment in a general hospital.

Treatment is conducted upon general lines, rest, feeding, indoor and out-door recreation, and massage in suitable cases. At the Maghull Military Hospital a form of psycho-analysis has been used with benefit in selected cases. The results of treatment at Maghull show about 40 per cent. of cases returned to light duty.

3. The Mental Hospitals-Napsbury War Hospital and Dykebar War Hospital, N.B.-These hospitals receive the majority of their patients from D Block, Netley; but Napsbury admits mental cases also from military hospitals in the Southern Command and the Midland counties; Dykebar admits also from military hospitals in the Scottish Cornmand and the northern counties. The Napsbury War Hospital is the hospital section of the parent asylum ; Dykebar is one of the most recent of the Scotish asylums built upon the villa pattern. The available accommodation in the two hospitals is about 700 beds.

The patients transferred to these hospitals are of a certifiable type and incluae most of the severe forms of acute mental disorder - the confusional psychoses, mania, the graver melancholias, acute delusional and hallucinatory psychoses, dementia præcox, mental deficiency with confusion, general paralysis of the insane, and epilepsy with mental symptoms. In accordance with accepted policy, none of the patients in these hospitals is certified as a person of unsound mind. Each patient is given a reasonable period of treatment with a view to recovery. In consequence, however, of the accumulation of chronic and incurable cases which was observed a few months ago, it was decided to board and discharge to asylums all cases of general paralysis of the insane, of epilepsy with insanity, and all patients who had been in asylums prior to enlistment. A certain number of chronic cases also are boarded and discharged to asylums if no improvement is recorded after a fair and reasonable period of observation and treatment.

It is obvious from the nature of the disorders that the percentage of cases returned to light duty must be small, but the figures from the Napsbury War Hospital show from 10 to 15 per cent. of cases discharged to light duty.

\section{CONGESTION IN THE TREATMENT OF CASES OF EPIDEMIC CEREBRO- SPINAL MENINGITIS.}

\author{
By DUNCAN FORBES, M.D. EdIN,, D P.H. CAMB., \\ MEDICAL OFFICER OF HEALTH, BRIGHTON; \\ AND
}

EVELINE COHEN, F.R.C.S. IREL., RESIDENT MEDICAL OFFICER, BRIGETON ISOLATION HOSPITAL.

THE treatment advocated in this short paper is simple and does not interfere with concurrent treatment. Congestion of the cerebral vessels is brought about by raising the foot of the bed not simply on the usual ward blocks, but on stools or lockers, so that the bed and the patient's body, no pillow being allowed, make an angle of from $14^{\circ}$ to $23^{\circ}$ with the floor. That the method influences the course of the disease profoundly will be gathered from a study of the five cases reported.

Everyone who has treated this disease can call to mind cases which seemed to all but get over the illness, but became chronic and died. It was in this type of case that treatment by congestion was at first tried, later congestion was found useful in the earlier stages of the disease. The histories of the first five cases treated are given in detail.

CASE 1.-Male, aged 20. The patient suffered from a mild attack of the disease and recovered some degree of forward movement of the head. Unfortunately, although he felt well, a slight stiffness of the neck persisted, his temperature was irregular, and leucocytes, chiefly lymphocytes, were present in a clear spinal Ifuid containing 0.1 per cent. of albumin. On the twenty-seventh day of illness, at $11 \mathrm{~A} . \mathrm{M}$. (see Chart), the lower end of the bed was raised on lockers (angle 23). In the evening of the same day the temperature rose only to $99.6^{\circ} \mathrm{F}$. and thereafter remained normal. The bed was lowered four days after the temperature had fallen to normal.

Summary.-A mild case with immediate fall of temperature and complete recovery following the raising of the foot of the bed.

CASE 2.- Male, aged 20. The patient on the eighteenth day of illness vomited, his head was retracted, and he had every appearance of becoming a chronic case (see Chart). On puncture on the nineteenth day the pressure was found to be low. The foot of the bed was raised on stools (angle 14\%). There was occasional vomiting until the twenty-second day, when the patient complained of very severe headache and the bed was lowered. On the twenty-third day on puncture a hazy fluid at high pressure was withdrawn. On the twenty-fourth day the patient felt quite well, and the temperature fell to normal. On the twenty-fifth day his. head was freely movable. Thereafter his recovery was uninterrupted.

Summary.-Case becoming chronic, spinal fluid at low pressure, foot of bed raised to angle of $14^{\circ}$; after three das congestion caused severe headache, accompanied bv high pressure of the spinal fluid. On lowering of bed a rapid recovery.

CASE 3.-Male, aged 21. After three weeks' illness the patient continued to have marked reck stiffness and temperature (see Chart), and there was no diminution in the number of the diplococci in the spinal fluid. On the twentythird day of illness the foot of the bed was raised on blocks (angle $9^{\circ}$ ). No improvement followed, so the bed was raised on stools (angle $14^{\circ}$ ) on the twenty-fifth day. The temperature fell the same evening, but there was still neck stiffness On the twenty-seventh day, the temperature again having risen, the bed was further raised on lockers (argle 23\%). The temperature gradually fell and was normal on the thirtieth day. On the thirty-first day the patient felt well and his head wás freely movable. As the patient continued to feel well his bed was unfortunately lowered to blocks (angle $9^{\circ}$ ) on the thirty-third day at 10 A.M. In the evening of the same day the temperature rose to $102^{\circ} \mathrm{F}$. and he had a slight headache. On the thirty-fourth day his bed was again raised on lockers. By the thirty-eighth day the temperature was normal, the patient felt well, but some stiffness of the neck remained. As the slowness of cure-was attributed to the patient raising his head and lying on his side he was restrained from doing anything except lying on his back from the evening of the thirty-eighth day. His temperature rose on the following day, and on the fortieth day reached $102 \cdot 2^{\circ}$ at 6 A.M. Vomiting began at 3 A.M. on the same day, and as he could retain nothing his bed was lowered at 9 A.M. The romiting ceased at 4 P.M. On the forty-second day the temperature was normal, and he felt well on the forty-third day, but some slight neck stiffness persisted. On the evening of the forty-fourth day, apparently 


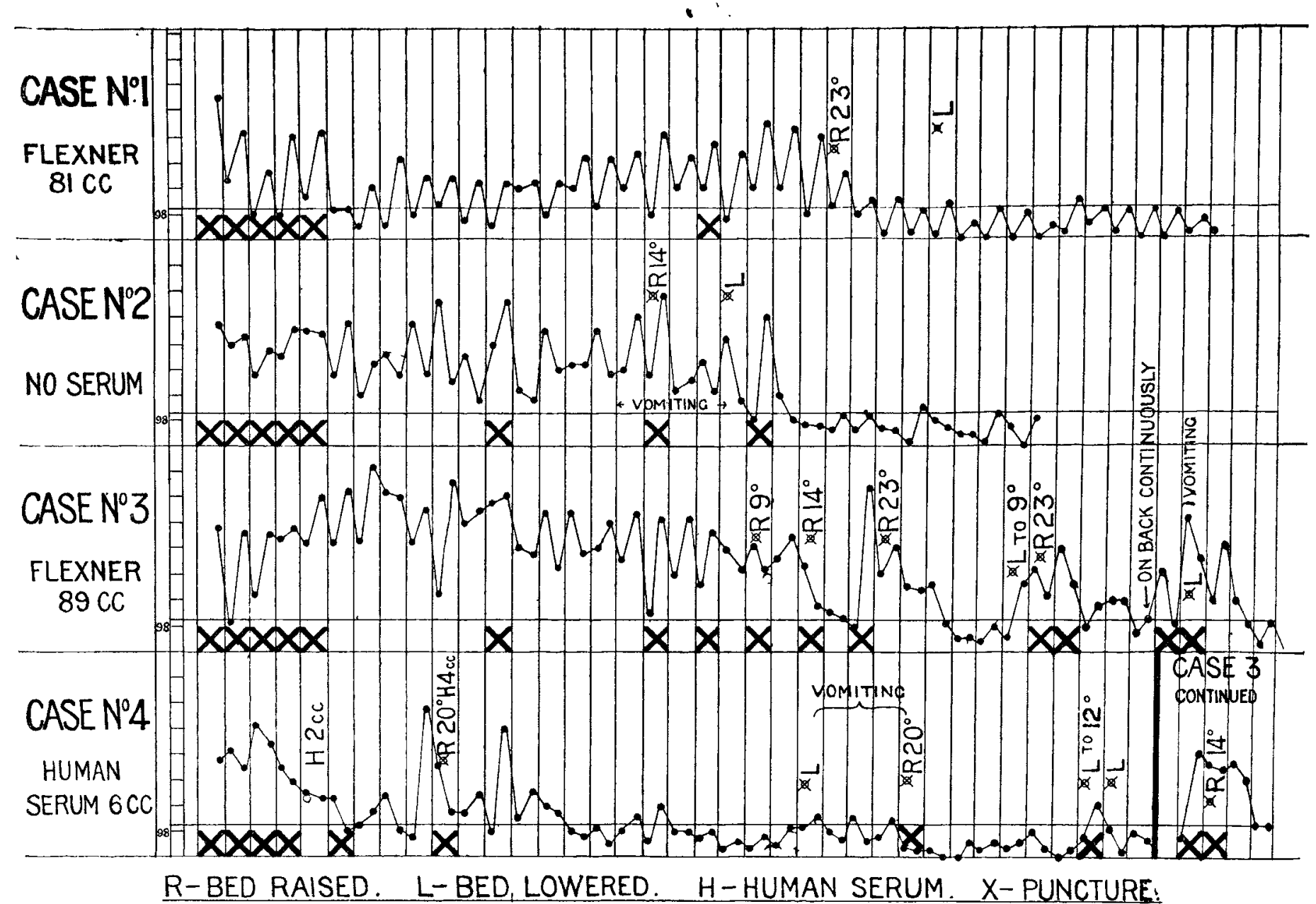

following puncture early on the same day, the temperature rose to $101 \cdot 6^{\circ}$, and there were headache, backache, and later vomiting. On the forty-fifth day his bed was raised to $14^{\circ}$. By the forty-seventh day his temperature had reached normal. His bed was gradually lowered from the fifty-fifth day and the patient has remained well.

Summary.-No improvement following a slight raising of the foot of the bed, free movement of the head and a normal temperature following the raising of the bed on lockers. A relapse immediately after the too early lowering of the bed. Some improvement follows a partial application of the treatment, with a marked reaction following its stringent application; this too great congestion is followea by a rapid improvement on the lowering of the bed. A relapse follows puncture. Final recovery after a prolonged raising of the foot of the bed to angle of $14^{\circ}$.

CAsE 4. - Male infant, aged 7 months. This was a peculiarly interesting case. Puncture alone was tried, as indicated on the chart. The spinal fluid contained large numbers of organisms, both intra-and extra-cellular. As a last resort, on the morning of the sixteenth day of illness 2 c.c. of the serum ${ }^{1}$ of a convalescent patient were injected intrathecally. The effect was astonishing, as the infant, who had required nasal feeding, resumed taking the bottle in the afternoon and seemed much better. The temperature gradually fell and was normal on the evening of the eighteenth day. The fluid at that time was hazy and showed few diplococci as compared with the large number previously present. Unfortunately the child became worse, his colour again became bad on the nineteenth day, and he vomited. He became progressively worse until the twenty-second day of illness, when a further 4 c.c. of the serum of the same con valescent was given and the cot was left raised at an angle of $20^{\circ}$. On the twenty-third and twenty-fourth days there was vomiting, but on the twenty-sixth day he seemed better, and on the twenty-seventh day had a normal temperature. The child remaining quite well the cot was lowered on the thirty-sixth day at 10 A.M. At 2.30 P.M. there was vomiting. Thereafter the patient became fretful and vomited two or three times a day up to the fortieth day, when he was punctured and showed a clear fluid containing a few lymphocrtes. The cot was again raised to an angle of $20^{\circ}$ and he almost at once seemed to be better and comfortable. There was no further vomiting. The fluid being normal, the bed was lowered to blocks on the forty-seventh day, and finally lowered altogether on the forty-eighth day. The recovery was uninterrupted.

1 The injection of 10 c.c. of human anti-serum in a later case was followed by shock and all the symptoms of anerum in a later case was con lition was rendered much worse.

Summary.-A marked reduction in the number of organisms following the intrathecal injection of the serum of a convalescent. The number of organisms remain scant but the child becomes worse. A further injection of human serum, and the end of the cot left raised is followed by a normal temperature. The lowering of the cot later leads to fretfulness and vomiting ; these at once stop when the cot is again raised. Eight days later the cot is lowered and the child remains well.

CASE 5.-Male, aged 22. This patient was admitted on the second day of illness with a normal temperature. The upper end of the bed was raised to an angle of $14^{\circ}$. During the next few days, although the temperature remained normal and the headache became less severe, the neck was stiffer and retraction of the head developed. On the fifth day the lower end of the bed was raised to $14^{\circ}$. In the evening the temperature, which had remained normal, rose to $99^{\circ} \mathrm{F}$. Improvement set in and continued thereafter.

Summary.-A subacute case with rise of temperature and cure following the raising of the lower end of the bed.

From a consideration of these and further cases we feel sure that the method deserves a further and more extended trial. It would appear that continued raising of the foot of the bed leads to congestion of the cerebral vessels. In mild cases in a few days a normal temperature and free movement of the head result, and the recovery is uninterrupted. In more severe cases the temperature rises and the patient more gradually recovers, the recovery being at first accompanied either or both by increased tension of cerebro-spinal fluid and a greater migration of polymorphs. If the foot of the bed has been raised too high there may be very severe headache and persistent vomiting due to a too reat congestion and its results. In such cases, if the bed is lowered and the tension is relieved by puncture, the patient yradually recovers. As different cases require varying degrees of stimulation, no hard-and-fast rule can be laid down as to the height to which the foot of the bed should be raised.

Although at first this method of treatment was only tried in cases which threatened to become chronic, we recently have had good results from its application in the early stages of the disease. On admission the last two cases, after being punctured, were given morphia and the lower ends of the beds were raised on lockers during the day, being lowered or partially lowered at night. One of the patients who was admitted violently delirious recovered free movement of the neck in three days, the other, a serum-treated 
case, is still ill. Whilst experience may show that it is best to raise the foot of the bed on lockers early in the disease, in more chronic cases the bed should be raised first on blocks and rapidly higher until the patient begins to show a marked reaction or a more freely movable neck. At that point heightening should stop and the patient be allowed gradually to recover, the foot of the bed being left continuously raised until some seven days after apparent recovery, and thereafter gradually lowered. Other points worthy of note are that patients should lie on their backs as much as possible during treatment, and should have no pillows. When there is marked retraction and the patient cannot lie on his back the bed should be tipped sideways and the patient's head be allowed to hang over the lower edge. This method is particularly useful for children, but as it is somewhat drastic it has not been practised for more than two hours at a time. It is always a serious mistake to puncture a patient who is progressing towards recovery, or who bas apparently recovered.

This paper is in the nature of a preliminary note; its object is not only to bring to the notice of the profession a valuable method of treatment, but also to enlist its aid in finding out how the method can most usefully be applied in practice.

\section{THE TREATMENT OF GUNSHOT WOUNDS BY PACKING WITH SALT SACS.}

BY ALFRED J. HULL, F.R.C.S. ENG., MAJOR, ROYAL ARMY MEDICAL CORPS, BRITISH EXPEDITIONARY FORCE, FRANCE.

THE treatment of septic wounds by a pack which is allowed to remain in the wound for several days is so opposed to the usual teachings of surgery that at first sight its utility may be doubted. Nevertheless, such treatment has proved one of the most effective methods of dealing with septic wounds during the present war. It has also been found to be one of the most generally applicable procedures for the treatment of secondary hæmorrhage. It possesses the great advantage of simplicity, an important factor when large numbers of cases require the attention of a limited staff. It also allows transport to be undertaken without any anxiety regarding the dressing of the wound.

When it is necessary to drain a wound cavity, three methods are available. The ideal method would be to expose the whole of the interior of the wound so that its walls become exposed and more or less flat. There are anatomical and surgical objections to this as a rule, but it is occasionally employed, for example, when a rectal fistula is slit up when an amputation is performed by the guillotine method, and in certain cases of excision of wounds. Very inferior to this ideal method is the drainage of every pocket and cavity of a wound by drains. The interior of the wound is often very irregular, and it is sometimes impossible to ensure that every pocket is drained. The third method, treatment by solid salt, imitates the first or open method, by draining every portion of the surface of the interior.

The treatment of septic wounds must fulfil certain requirements. The infecting microbes must be removed by some form of lavage. The outflow of lymph must be increased. The bacterial growth must be repressed and the coagulation of the discharge must be prevented. The tissues of the wound must not be damaged either by chemical or mechanical measures. It is common ground that free exposure of the infected tissues must be made in order that adequate drainage be obtained, but many opinions are held as to the correct method of dressing the wound. These requirements may be met by the use of hypertonic solution of common salt.

It can be demonstrated experimentally that the higher the concentration of salt the greater will be its power of attracting water and albuminous substances. The "drawing" power of salt has been known for many years. Sir A. E. Wright's original lymphagogic solution consisted of a 5 per cent. solution of common salt mixed with 0.5 per cent. solution of sodium citrate. Early in the present war Sir A. E. Wright recommended the hypertonic solution of salt for the purpose of lavage and repression of the bacterial organisms of septic wounds. Colonel C. B. Lawson invented tablets of compressed salt which he placed in the lumen of drainage-tubes. Colcnel Gray, C.B., originated a modification of Sir A. E. Wright's hypertonic treatment by introducing the use of solid salt in the form of tablets. The tablets wrapped in gauze formed a solution of salt.

Packing wounds with gauze impregnated with concentrated salt solution must not be confused with the plugging of wounds with ordinary dressings. Wound cavities are only potential spaces, and in order to drain them the walls must be kept apart. Ordinary drainage-tubes rarely drain every recess, and elaborate methods are required to obtain drainage from every portion of the walls and avoid the shutting off of pockets. A wound cavity filled with gauze and salt may be considered as effectively drained as if the cavity were inverted and the walls dressed as a surface wound. Every portion of the surface area is drained by the osmotic action of the salt, and the capillary action of the gauze and bandage is continually removing the discharge and producing a continuous and automatic drainage and lavage of every portion of the interior of the wound.

The tablets of salt must not come in contact with the tissue or sloughing will often ensue. The wall of the cavity must be well protected by gauze. The solid salt sac is an improvement upon the tablets. It consists of a twowalled sac of suitable size made of bandage, between the layers of which four layers of gauze are placed. The interior of the sac is filled with salt and the tail of the bandage forms a drain.

The sac is made by laying a strip of open woven bandage about a foot in length flat on the table. On this strip four layers of gauze are placed the same width as the bandage but about 4 inches in length. On top of the gauze another strip of bandage is placed. The sides of the bandage strips are then brought together by doubling the bandage in its long diameter and sewing to form a sac. The sac is filled with salt, a few stitches are put in to close the mouth. These sacs are made in sereral sizes, sterilised in an autoclave, and stored ready for use. One or more of them are used to pack the wound, the intervals between the sacs being filled with gauze. A tube of perforated zinc or rubber may be passed into the depth of the wound in cases of large septic wounds. Long slender sacs are made to fill bullet tracks. Small sacs are made for draining tracks in the brain. The free end of the sac is passed into a vessel containing saline solution and the wound then drains by capillary attraction. A fine rubber tube may be run in along each sac, and drip irrigation carried out if required. The salt remains undissolved for several days and when the sacs are removed from a wound at the end of a week undissolved salt still remains.

The frequency with which wounds are dressed very often depends upon empirical convention rather than on a scientific or clinical reason. Wounds are often dressed without any clear idea of the reason for removing the dressings. A dressing sticking to a wound is frequently torn off, tearing delicate granulation tissue, undoing the healing of the wound, and exposing raw surfaces for septic absorption. A more unscientific treatment cannot be imagined. In some cases it is said, the wound is dressed in order to remove the discharge. This is simply an admission that the dressing has been acting like a cork in a bottle. A rise of temperature is another reason given for removing a dressing, but if the wound is being properly treated, changing the dressing will not lower the temperature.

There are two ways of dressing a wound so that no harm is done by the changing of dressing: one is to keep a light dressing continually moist so that its remoral is not felt by the patient and no tissue is damaged; the other is to leave the dressing until it is quite moist and can be removed without pain. The dressing of deep wounds is too often merely a ritual, the dressings and drainage-tubes are removed and replaced by clean ones, but the complicated series of recesses which make up the interior of most wounds cannot be reached. They may be reached by irrigation, but some portion of the wound will escape the dister,ding effect of a drainage-tube, and the walls will fall together and a pocket will result.

The effect of the salt sac is to form a concentrated solution of salt which promotes the resolution of inflammatory induration and aids the separation of dead tissue by solution of coagulated lymph. A powerful outgoing stream of healthy lymph is produced which carries microbes and débris out of the wound and retards the bacterial growth within it. It is unnecessary and undesirable to continue the action of this hypertonic solution of salt once the wound becomes clean. If the wound is clean the sacs are removed 